\title{
Plasminogen activator inhibitor-1 is locked in active conformation and polymerizes upon binding ligands neutralizing its activity
}

\author{
JERZY JANKUN ${ }^{1-3^{*}}$, ZOFIA SPECHT $^{1,2}$, MARIA SZKUDLAREK $^{1,2}$, ROBERT GREENFIELD $^{5}$, \\ BHAVNA GAIKWAD ${ }^{5}$, LATCHEZAR TRIFONOV ${ }^{6}$, JEAN VAUGEOIS ${ }^{6}$, \\ ANSARI M. ALEEM ${ }^{1,2}$, VENKATESHA BASRUR ${ }^{4}$, STEVEN H. SELMAN ${ }^{1-3}$, \\ MARIA I. ZAVODSZKY ${ }^{7}$ and EWA SKRZYPCZAK-JANKUN ${ }^{1,2^{*}}$
}

\begin{abstract}
${ }^{1}$ Urology Research Center, ${ }^{2}$ Department of Urology, ${ }^{3}$ Department of Physiology and Molecular Medicine, and ${ }^{4}$ Program in Bioinformatics and Proteomics/Genomics, Department of Microbiology and Immunology, Medical University of Ohio, Toledo, OH 43614-5807; ${ }^{5}$ American Diagnostica Inc., Stamford, CT 06902, USA; ${ }^{6}$ Quebepharma Recherche Inc., Montreal H2X 3Y8, Canada; ${ }^{7}$ Department of Biochemistry, Michigan State University, East Lansing, MI 48824-1319, USA
\end{abstract}

Received October 20, 2005; Accepted November 18, 2005

\begin{abstract}
Plasminogen activator inhibitor-1 (PAI-1), a member of the serpin super-family, forms a covalent complex with its target proteinases, such as tissue and urokinase plasminogen activators. Thus, PAI-1 controls the physiological and pathological proteolysis. An abnormal expression of PAI-1 has been observed in different diseases, which can be treated by returning the proteolysis back to normal physiological levels. It has been reported that some PAI-1 inhibitors neutralize its activity by accelerating the conversion of PAI-1 into a latent form. We have found small organic chemicals that also neutralize PAI-1 activity, but by a different mechanism. Using the NBD fluorescent probe [N,N'-dimethyl-N-(acetyl)-N'-(7nitrobenz-2-oxa-1,3-diazol-4-yl)] incorporated into the reactive center loop (RCL) of PAI-1, we measured the kinetics of conversion from an active to a latent form. Unexpectedly, we found that some inhibitors of PAI- 1 arrest this serpin in its active form instead of increasing the speed of conversion. Using docking calculations, we located two possible binding sites for these chemicals. The sites are in proximity of the $\mathrm{P} 1 / \mathrm{P} 1$ ' amino acids of the RCL of PAI-1. Binding in this area can inactivate PAI-1 and additionally create a steric obstacle on the RCL making insertion of this loop between the A3 and A5 strands more difficult; hence abolishing a necessary step in the conversion of this protein into the latent form. Additionally, PAI-1 inhibitors link the RCL of one PAI-1 molecule with the
\end{abstract}

Correspondence to: Dr Jerzy Jankun, Urology Research Center, Medical University of Ohio, 3065 Arlington Ave., Toledo, OH 43614, USA

E-mail: jjankun@meduohio.edu

${ }^{*}$ Contributed equally

Key words: PAI-1, inhibitors, polymerization, cancer, molecular modeling strand $3 \mathrm{C}$ and strand $4 \mathrm{C}$ or helix $\mathrm{A}$ and strand $1 \mathrm{~B}$ regions of the other PAI-1 molecule aiding polymerization or stabilizing the junction of the two. The polymerization of PAI-1 reduces PAI-1 activity by encapsulating the critical RCL fragment inside the formed PAI-1/ PAI-1 polymers.

\section{Introduction}

Plasmin-driven proteolysis plays an important role in normal physiological and pathological processes. Plasminogen, upon activation into its active form called plasmin, can hydrolyze a broad spectrum of proteins. Also, plasmin could activate other proteolytic enzymes, triggering further plasmin-initiated proteolysis. Activation of plasmin occurs through proteolytic cleavage of plasminogen by its activators. Two of the most important activators are the urokinase plasminogen activator (uPA) and tissue plasminogen activator (tPA). Plasmin proteolysis is also regulated by the inhibition of plasminogen activators. Four basic types of PA are known: plasminogen activator inhibitor-1 (PAI-1), -2, -3, and protein nexin (1-4). It seems that PAI-1 plays a dominant role in different diseases and has been extensively studied by many laboratories $(5,6)$.

Plasmin-driven fibrinolysis plays a critical role in a variety of different diseases. The interaction between tPA and uPA and its inhibitors affect the fibrinolytic activity of human blood. In addition to fibrin degradation, the fibrinolytic system components alter several biological events such as angiogenesis, tumorigenesis, arteriosclerosis and cellular migration (7). An abnormally high expression of PAI-1 has been reported in various human diseases, including atherosclerosis, coronary heart disease, sepsis, renal and lung fibrosis, obesity and insulin resistance $(6,8)$. Furthermore, an overexpression of PAI-1 has been demonstrated in atherosclerotic artery plaque compared with normal vessels (9).

PAI-1 is also considered one of the key regulators of tumor invasion and metastasis, as well as cancer-related angiogenesis. One of the hallmarks of cancer progression resides in the capacity of cells to cross through several tissue boundaries. 
Degradation of the cell microenvironment, consisting of matrix macromolecules, is not precisely controlled in carcinogenesis, unlike in normal physiological conditions. To achieve tissue penetration, cancer cells stimulate their proteinase machinery and overproduce and bind proteases, which permits cell migration through a degraded extracellular matrix (10). One of these proteases is urokinase plasminogen activator. It has been shown that inhibition of uPA activity reduces metastasis in in vitro and in vivo models $(11,12)$. Additionally, during carcinogenesis, the advancing tips of capillary angiogenic vessels express a high activity of uPA. Inhibition of uPA activity results in a reduction of angiogenesis and cancer size as shown in in vivo models of the breast, colon, prostate and many other cancers (12-14). It has been proposed by us and others that pathological plasminogen activation system upregulated proteolysis could be eradicated by PAI-1 mutants with an extended half-life (14-17).

PAI-1 is a representative of the serpins, which are members of the super-family of serine protease inhibitors sharing many structural and biochemical similarities. Inhibitors of serine proteases act by making a 1:1 stochiometric complex by inserting P1 Arg into the protease's specificity pocket, followed by the formation of a covalent bond between the enzyme's catalytic site and the residues at the reactive center loop. PAI-1 is not a stable molecule and converts itself into the latent form with a half-life in the range of $t_{1 / 2}=1-2 \mathrm{~h}$. This conversion is associated with the partial insertion of the reactive loop (P4-P10') inside the A B-sheet of PAI-1 molecule. In such a conformation, P1-P1' and other sites are not accessible for reaction with uPA or tPA. Several mutants have been produced to reduce or prevent the insertion of the reactive loop into the PAI-1 molecule. Tucker et al produced a 'GATE' mutant with an extended half-life $t_{1 / 2}=6 \mathrm{~h}$ by a single point mutation (18). Also, the Ginsburg group produced mutant 1B14 with an even longer half-life by four point mutation (Lys 154 $\rightarrow$ Thr, Glu319 $\rightarrow$ Leu, Met354 $\rightarrow$ Ile, Asn 150 $\rightarrow$ His) $(19,20)$. We have produced several PAI-1 mutants by replacing chosen amino acids with cysteine in hopes of creating disulfide bridges, which could make this insertion more difficult. This resulted in proteins with an extended half-life of serpin activity from 2 to $>700 \mathrm{~h}$ depending on the mutant (15). These genetically engineered PAI-1s were investigated as therapeutics on animal models $(14,15)$. Mutations in these novel proteins are in the regions around $\alpha$-helices $\mathrm{D}$ and $\mathrm{E}$ and $\mathrm{B}$-strands $1 \mathrm{~A}$, $3 \mathrm{~A}$ and $5 \mathrm{~A}$, which has been reported to be critical for the conversion of PAI-1 into its latent form (15,18-20).

The various biological effects of PAI-1 create a dilemma. On the one hand, local PAI-1 overexpression can reduce cancer growth. On the other hand, increased PAI-1 can enhance fibrin accumulation and cause a stroke or other ailments. Thus, balancing plasmin driven proteolysis at a level that cures different diseases requires the inhibition of abnormal uPA/ plasmin activity, which can be controlled by mutated PAI-1 or by inhibiting PAI-1 activity to prevent reaching pathological levels. While inhibition of uPA- or tPA-initiated proteolysis in the disease was studied extensively, inhibition of PAI-1 has drawn much less attention (1,21-23). Therefore, we investigated several PAI-1 inhibitors to understand their mechanism of action with the ultimate goal of developing future pharmaceuticals neutralizing PAI-1 activity.

\section{Materials and methods}

Plasminogen activator inhibitors. Wild PAI-1 and the fluorescent labeled PAI-1 variant (NBD P9 PAI-1) conjugated with the fluorescent tag N,N'-dimethyl-N-(acetyl)-N'-(7nitrobenz-2-oxa-1,3-diazol-4-yl) at the reactive center loop were purchased from Innovative Research Inc., Southfield, MI $(24,25)$.

Inhibition of PAI-1 activity. Inhibition of PAI-1 activity toward uPA was analyzed by microtiter assay, which measured the ability of a compound to block PAI-1 inhibition of uPA proteolytic activity. A stock solution $(10 \mathrm{mM})$ of each compound and its dilutions were prepared in DMSO. Twenty microliters of $6 \mathrm{nM}$ wild-type human PAI-1 was incubated for $20 \mathrm{~min}$ at room temperature with $52 \mu \mathrm{l}$ of $50 \mathrm{mM}$ HEPES, $150 \mathrm{mM} \mathrm{NaCl}, 0.05 \%$ Tween-20, 1\% BSA, pH 6.6 buffer and $8 \mu \mathrm{l}$ of test compounds (from $10 \mathrm{nM}$ to $100 \mu \mathrm{M}$ final concentration). Twenty microliters of $8 \mathrm{nM}$ high molecular weight uPA (American Diagnostica Inc.) in $0.05 \mathrm{M}$ Trisbuffered saline $\mathrm{pH} 8.4$ was added to each well and the mixture was incubated for $5 \mathrm{~min}$ at room temperature. Fifty microliters of $0.1 \mathrm{mM}$ Spectrozyme UK fluorogenic substrate (American Diagnostica Inc.) was added to each well and uPA proteolytic activity was monitored for $30 \mathrm{~min}$ at $37^{\circ} \mathrm{C}$ on a SpectraMax (Molecular Devices Inc.) spectrofluorometric microplate reader. The excitation wavelength was set at $360 \mathrm{~nm}$ and emission wavelength was set at $440 \mathrm{~nm}$. Kinetic data were analyzed using Softmax Pro software. The difference between the UPA activity in the presence or absence of untreated PAI-1 was the total PAI-1 activity $(100 \%)$. The PAI-1 inhibitory activity of the compounds was expressed as a percentage of the total PAI-1 activity. Controls were performed to ensure the absence of any direct effect of the test compounds on uPA activity alone.

PAI-1 small molecule inhibitors. E30C (1,7-bis-[(4-hydroxy3-methoxy-5-phenylsulfinyl)phenyl]-1,6-heptadiene-3,5dione), E57C (1,7-bis-\{[4-hydroxy-3-methoxy-5-(4-methyl1-piperazinyl)methyl]phenyl $\}$-1,6-heptadiene-3,5-dione), L265 ((2S)-3-(1-benzothien-3-yl)-2-\{[(5-bromopyridin-3-yl) carbonyl]amino\} propanoic acid), LT21B 5-[(t-Butyloxycarbonyl)amino]-2-hydroxy-N-(2-hydroxyethyl)benzamide, LS12 (5-[(Z)-(1-benzyl-2,5-dioxoimidazolidin-4-ylidene) methyl]-2-hydroxybenzoic acid) were synthesized in Quebepharma Recherche Inc., Montreal, Canada. The curcuminoids (E30C, E57C) were prepared following Pabon's procedure as described previously (26-28). The bis-phenylsulfinyl curcumin, E30C, was purified by silica gel chromatography using chloroform:ethyl acetate: acetonitrile: acetic acid = 95:2.4: 2.4:0.2, 92:3.8:3.8:0.4, 70:14.4:14.4:1.2, and 48:25:25:2. E57C was purified by silica gel column chromatography using chloroform:ethanol (gradient ethanol from 0 to $20 \%$ ).

The amide, L265, was prepared by acylation of 3-(thianaphthen-3-yl)-L-alanine with 5-bromonicotinic acid chloride in water at $0^{\circ} \mathrm{C}$ under nitrogen at $\mathrm{pH} 11$. The reaction mixture was extracted with diethyl ether, the water layer was acidified to $\mathrm{pH} 2.0$, the solid product was filtered, washed with water and dried. No further purification was needed since the ${ }^{1} \mathrm{H}$ NMR spectrum showed this to be pure L265. For the synthesis 
of LT21B, 5-aminosalicylic acid was first protected as BOCderivative, which was then converted to active succinimido ester. The crude active ester was reacted with 2-aminoethanol at room temperature for $48 \mathrm{~h}$. Ethyl acetate was added and the organic layer was washed with water, dried and the solvents removed under vacuum. The crude product was recrystallized from ethyl acetate:hexane (2:3) to provide pure LT21B.

For the preparation of LS12, a suspension of 5-formylsalicylic acid (2 mM), 1-benzylhydantoin $(2 \mathrm{mM})$ and 2-aminoethanol $(360 \mathrm{mg})$ in water $(10 \mathrm{ml})$ was stirred at $90^{\circ} \mathrm{C}$ for $4 \mathrm{~h}$. The reaction mixture was cooled to room temperature, acidified with $5 \mathrm{~N} \mathrm{HCl}$, filtered and washed with water to provide pure LS12 as a colorless solid. The purity of all compounds was $\geq 98 \%$ as determined by ${ }^{1} \mathrm{H}-\mathrm{NMR}$.

Inhibitors were dissolved in DMSO $(\sim 10 \mathrm{mg} / \mathrm{ml})$ and added to the PAI-1 solution ( $2 \mathrm{nM})$ in $0.01 \mathrm{M}$ phosphate-buffered saline $\mathrm{pH} 7.4$ and incubated for up to 24 , or in some cases, 96 h. Fluorescence was measured at 5, 30, 120, 300, 420, $660,1,440$ and, in some cases, 5,760 min after initiating the reaction of PAI-1 NBD with inhibitors. PAI-1 NBD incubated with DMSO served as a control. Measurements were performed at least in duplicate.

The kinetics of RCL insertion. The kinetics of reactive center loop (RCL) insertion of NBD P9 PAI-1 between A3 and A5 $\beta$-strands was performed by monitoring an increase in NBD fluorescence emission in the range of 500-590 nm, excitation 480 nm, using spectrofluorometer Mark I, Farrand Optical Co., Inc., New York, NY. The fluorescence emission of NBD P9 PAI-1 was enhanced 4- to 7-fold upon insertion of the RCL into $B$-sheet $A$ and the peak centered at $542 \mathrm{~nm}$ for active PAI-1 was blue-shifted to $530 \mathrm{~nm}$ upon RCL insertion and conversion into an inactive form $(24,25,29,30)$.

Non-reducing gel electrophoresis. The electrophoresis was performed at room temperature in gradient gels with $4-12 \%$ polyacrylamide, in the absence of 2-mercaptoethanol. The following marker protein standards (See Blue Plus2 PreStained Standard from Invitrogen) were used: myosin $\left(M_{\mathrm{r}}\right.$ $191 \mathrm{kDa})$, phosphorylase $\left(M_{\mathrm{r}} 97 \mathrm{kDa}\right)$, BSA $\left(M_{\mathrm{r}} 64 \mathrm{kDa}\right)$, glutamic dehydrogenase $\left(M_{\mathrm{r}} 51 \mathrm{kDa}\right)$, alcohol dehydrogenase $\left(M_{\mathrm{r}} 39 \mathrm{kDa}\right)$, carbonic anhydrase $\left(M_{\mathrm{r}} 28 \mathrm{kDa}\right)$, myoglobin red $\left(M_{\mathrm{r}} 19 \mathrm{kDa}\right)$, and lysozyme $\left(M_{\mathrm{r}} 14 \mathrm{kDa}\right)$. Gels were stained with colloidal Coomassie blue (Invitrogen, LC 6025) or silverstain (31).

In-gel digestion with trypsin. The protein bands stained with Coomassie blue were excised from 4 to $12 \%$ gradient SDSPAGE gel destained with $30 \%$ methanol for $3 \mathrm{~h}$ at room temperature. In-gel proteolysis with modified sequencing grade trypsin (Promega, Madison, WI) was carried out essentially as described previously (32). Briefly, gel slices were further washed with $150 \mu 1$ of $50 \%$ acetonitrile in $0.1 \mathrm{M}$ ammonium bicarbonate buffer, $\mathrm{pH} 8.0$, for $30 \mathrm{~min}$. Gel slices were then diced into 1-mm cubes and dried using a vacufuge (Eppendorf). Trypsin $(0.5 \mu \mathrm{g}$, Promega) was added in a minimal volume of $0.1 \mathrm{M}$ ammonium bicarbonate buffer and the digestion was carried out for $16 \mathrm{~h}$ at $37^{\circ} \mathrm{C}$ with an additional aliquot of trypsin $(0.25 \mu \mathrm{g})$ added after $12 \mathrm{~h}$. Peptides were extracted once with $150 \mu \mathrm{l}$ of $60 \%$ acetonitrile containing $0.1 \%$ TFA for $30 \mathrm{~min}$, followed by a further extraction with $100 \mu \mathrm{l}$ of acetonitrile containing $0.1 \%$ TFA. All extracts were pooled and concentrated using Vacufuge to a final volume of $10 \mu 1$.

Protein identification by peptide sequencing using liquid chromatography-tandem mass spectrometer (LC-tandem MS). Two microliters of the digest were separated on a reverse phase column (Aquasil C18, $15-\mu \mathrm{m}$ tip x $75-\mu \mathrm{m}$ i.d. x 5-cm Picofrit column, New Objectives, Woburn, MA) using acetonitrile/1\% acetic acid gradient system (5-75\% acetonitrile over $35 \mathrm{~min}$ followed by $95 \%$ acetonitrile wash for $5 \mathrm{~min}$ ) at a flow rate of $250 \mathrm{nl} / \mathrm{min}$. Peptides were directly introduced into an ion-trap mass spectrometer (LCQ, ThermoFinnigan) equipped with a nano-spray source. The mass spectrometer was set for analyzing the positive ions and acquiring a full MS scan and a collision induced dissociation (CID) spectrum on the most abundant ion from the full MS scan (relative collision energy $30 \%$ ). Dynamic exclusion was set to collect 3 CID spectra on the most abundant ion and then exclude it for $2 \mathrm{~min}$. CID spectra were manually verified by comparing against an in silico tryptic digest of published PAI sequence using the MS-Digest and MS-Product provisions of Protein Prospector (http://prospector.ucsf.edu).

Docking of small organic molecules to PAI-1 using SLIDE. SLIDE is a docking/screening tool using distance geometry techniques to dock ligands into the binding site of the target protein via exhaustive matching of all possible ligand anchor fragments to all possible subsets of a template representing the protein binding site (33). The template consists of points identified as the most favorable positions for ligand atoms to form hydrogen bonds or make hydrophobic interactions with the neighboring protein atoms (34). The ligand anchor fragments were defined by triplets of interaction points, which are hydrogen-bonding ligand atoms or centers of hydrophobic atom clusters. Every combination of three template points were compared to every combination of three ligand interaction points in a search for complementary shape and chemistry, while regions outside the ligand anchor fragment (defined as the part of the ligand bounded by the current three interaction centers) were modeled flexibly. SLIDE models induced complementarity by making adjustments in the protein side chains and ligand upon binding, with the minimal set of necessary rotations determined by mean-field optimization (35). Each collision-free ligand orientation with at least $50 \%$ of its carbons buried was scored based on the number of hydrogen bonds and the hydrophobic complementarity with the protein.

The results from docking were scored independently by DrugScore (36). DrugScore calculates protein-ligand interaction energies employing a knowledge-based potential that reflects the frequency of pairwise atomic distances observed in protein-ligand complexes from the PDB.

Two molecules of PAI-1, chains A and C from PDB entry 1B3K (37), forming homodimer, were used as a target for predicting the binding orientation of all inhibitors tested by us. Grooves near the interface between chains $\mathrm{A}$ and $\mathrm{C}$ were defined as possible binding sites. After removing the points with less than 3 neighboring protein atoms, a template 

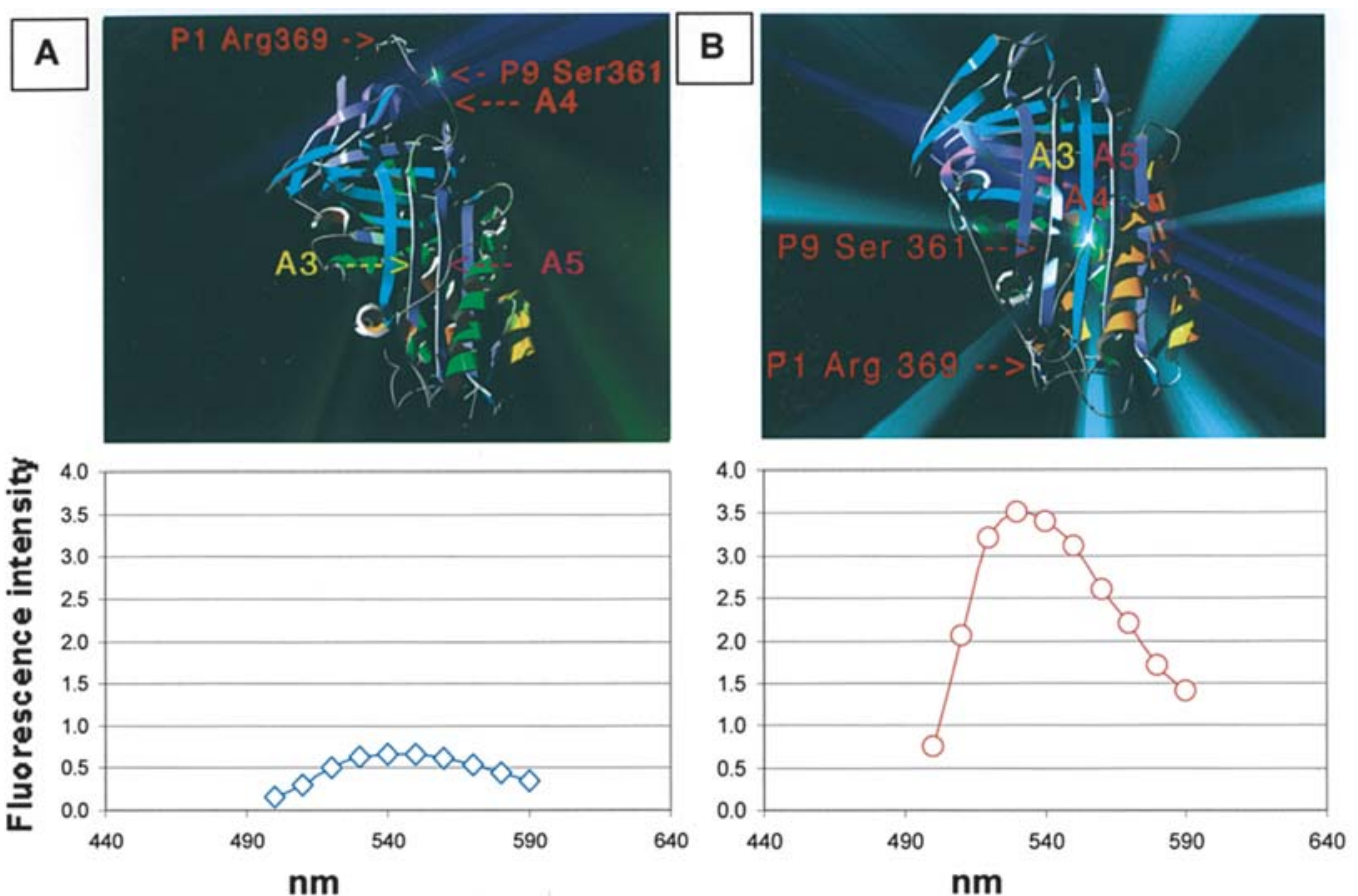

Figure 1. In NBD P9 PAI-1, Ser361 has been replaced with Cys in the P9 position and linked with NDB. A, active PAI-1 with extended RCL, position of strands indicated in yellow for A3 and in magenta for A5. What would be A4 in the latent form is indicated by an arrow. Fluorescence intensity of active PAI-1 is low as shown on the graph by the blue line and diamonds. B, in the latent form, RCL becomes the A4 strand (indicated in red) inserted between the A3 and A5 strand. The fluorescence intensity is 4-7 times higher than the active form as shown on the graph by the red line and circles. Atomic coordinates of active PAI-1 from PDB entry 1B3K (37), atom coordinates of latent PAI-1 from PDB entry 1C5G (18).
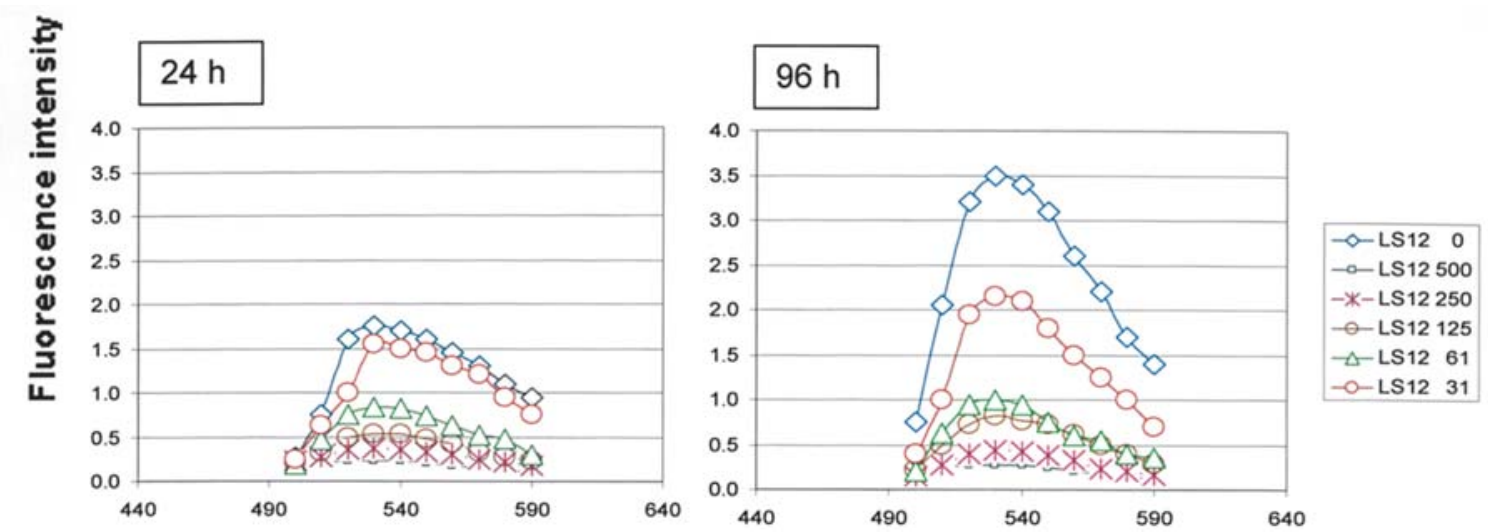

Figure 2. Emission spectrum of NBD P9 PAI-1 incubated for 24 and $96 \mathrm{~h}$ with various amounts of PAI-1 inhibitor (LS12 as an example, concentration in $\mu$ M).

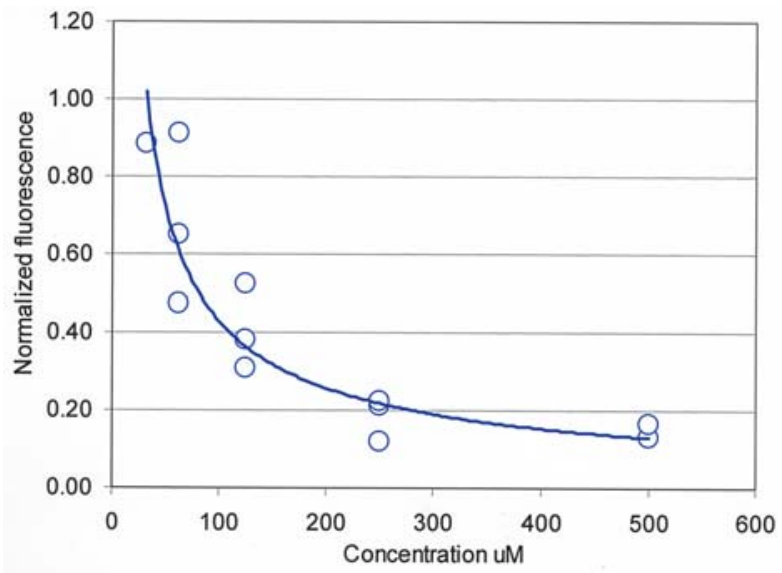

Figure 3. Concentration dependent normalized fluorescence of NBD P9 PAI-1 (triplicate measurements) incubated with different amounts of LS12. consisting of 38 hydrophobic and 49 hydrogen-bonding points was generated and used as an input for SLIDE. To ensure a thorough sampling of the ligand conformational space, the Omega program (OpenEye Scientific Software) was used to generate over 150 input conformers per inhibitor. Docked inhibitors were scored by SLIDE's own scoring function as well as by DrugScore to provide a list of top scoring orientations for each known inhibitor. We consider the most probable structure of a PAI-1-inhibitor complex to be the one with the highest score.

Molecular graphics. SwissPDB and Pymol viewers were used to display the three-dimensional structures of PAI-1 and to generate POV-Ray scenes (38). APBS tools of Pymol were used to evaluate the electrostatic properties of PAI-1 molecules (39). 
Table I. Concentration dependent capacity of PAI-1 inhibitors ${ }^{\mathrm{a}}$ to arrest PAI-1 in the active form expressed as $\mathrm{IC}_{50}$.

\begin{tabular}{|c|c|c|}
\hline Inhibitor & Structure & $\mathrm{IC}_{50} \mu \mathrm{M}$ \\
\hline LT21B & & $74.0 \pm 66.5$ \\
\hline LS12 & & $90.3+27.8$ \\
\hline E30C & & $106.8+63.4$ \\
\hline L265 & & $>500$ \\
\hline E57C & & \\
\hline & & \\
\hline
\end{tabular}

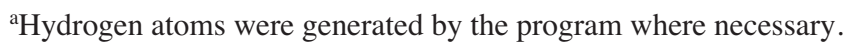

\section{Results}

The kinetics of NBD P9 PAI-1 conversion into the latent form. The kinetic characteristics of insertion of the RCL of NBD P9 PAI-1 between A3 and A5 B-strands in untreated solutions of PAI-1 followed the previously reported pattern $(24,25,29,40,41)$. As shown in Fig. 1A, the fluorescence of the active NBD P9 PAI-1 was weak, with a spectral maximum of $540 \mathrm{~nm}$ at the beginning of the experiment. When NBD P9 PAI-1 is converted into its latent form in a time-dependent manner, the RCL loop of the PAI-1 is inserted between A3 and A5 and causes a blue shift of the fluorescence spectra to $530 \mathrm{~nm}$ as well as an increase in the overall fluorescence intensity (Fig. 1B). After incubation of NBD P9 PAI-1 with the small molecule inhibitors (LS12 for example in Fig. 2), the rate of conversion of PAI-1 into its latent form was greatly reduced. As determined by the fluorescent spectral shift assay, the conversion of active NBD P9 PAI-1 to a latent form was not complete at up to $96 \mathrm{~h}$ (Fig. 2). This extended half-life of the active structural conformation of the NBD P9 PAI-1 was dependent on both the chemical structure of the small organochemical inhibitor as well as its concentration (Fig. 3 and Table I). Table I shows that, among the tested small organic compounds, which attenuate the conversion of serpin PAI-1 into its latent form, there was the following rank order of potency: L21B>LS12>E30C. Two others, L265 and E57C, showed only marginal activity at preventing the conversion of active PAI-1 into its latent form.

Inhibition of PAI-1 activity. As shown in Table II, with respect to the functional inhibition of PAI-1's interaction with uPA, the rank order of potency of the small molecule inhibitors is
Table II. Inhibition of PAI-1 activity by different organochemicals.

\begin{tabular}{lcc}
\hline Compound & Inhibitor concentration & \% PAI-1 inhibition \\
\hline E30C & $50 \mathrm{nM}$ & 60 \\
LS12 & $234 \mathrm{nM}$ & 54 \\
LT21B & $10 \mu \mathrm{M}$ & 25 \\
L265 & $1 \mu \mathrm{M}$ & 20 \\
E57C & E57C & E57C $^{\mathrm{a}}$ \\
\hline
\end{tabular}

${ }^{a}$ An intensely yellow compound that produced a fluorescence in the same wavelength as was used in the assay; therefore, its inhibitor PAI-1 activity could not be determined accurately.

E30C $>$ LS12>L21B. This effectiveness is in contrast to the previous experiment with the fluorescent spectral shift assay (Table I) which indicated a rank order of L21B $>$ LS12>E30C at arresting the PAI-1 in the active structural conformation. It is also noteworthy that, with respect to residual uPA as the endpoint, none of the tested compounds was able to completely inhibit PAI-1 (Table II). Although there were some limitations with utilizing higher doses of these compounds in the measurement of residual uPA, it is possible that these compounds are unable to completely inhibit PAI-1 or that they alter the affinity of PAI-1 for uPA but do not prevent them from interacting.

Polymerization of $w P A I-1$. It has been believed that PAI-1 does not form polymers, which was quite unique among active serpins (42). However, it has been shown recently that PAI-1 could spontaneously form polymers at low $\mathrm{pH}$ or polymerization could be induced by small organochemical ligands. Furthermore, polymerization is associated with partial inactivation of PAI-1 $(23,42-44)$. For that reason, we investigated whether the small molecule inhibitors that we selected could induce any polymerization of PAI-1. PAI-1 without any additives, with DMSO or inhibitors dissolved in DMSO, was incubated at $37^{\circ} \mathrm{C}$ for $30 \mathrm{~min}$ followed by incubation at $37^{\circ} \mathrm{C}$ or $70^{\circ} \mathrm{C}$ for $30 \mathrm{~min}$. We rationalized that the polymers formed should be preserved at $37^{\circ} \mathrm{C}$ and at least partially thermally dissociated at the higher temperature of $70^{\circ} \mathrm{C}$. As shown in Fig. 4, at $37^{\circ} \mathrm{C}$ dimers and trimers of different forms of PAI-1 could be observed on PAGE gels regardless of PAI-1 additives. The strongest bands for polymers were observed in LT21B> E30C. The rest of the inhibitors showed a smaller increase in polymerization ability. However, when samples were incubated at $37^{\circ} \mathrm{C}$ and subsequently warmed to $70^{\circ} \mathrm{C}$, polymerization was not detected only in the case of PAI- 1 without inhibitors and DMSO; presumably, because it had been thermally dissociated. Also, dimers of the active/RCC (RCC stands for reactive center cleaved) or active/latent form that were clearly visible in $37^{\circ} \mathrm{C} / 37^{\circ} \mathrm{C}$ incubation were not detected in all cases (Fig. 4). Nevertheless, all PAI-1 inhibitors showed strong bands of PAI-1 dimers and possible trimers (based on electrophoretic mobility). The strongest bands were observed in the case of LT21B >E30C $>$ LS12. 

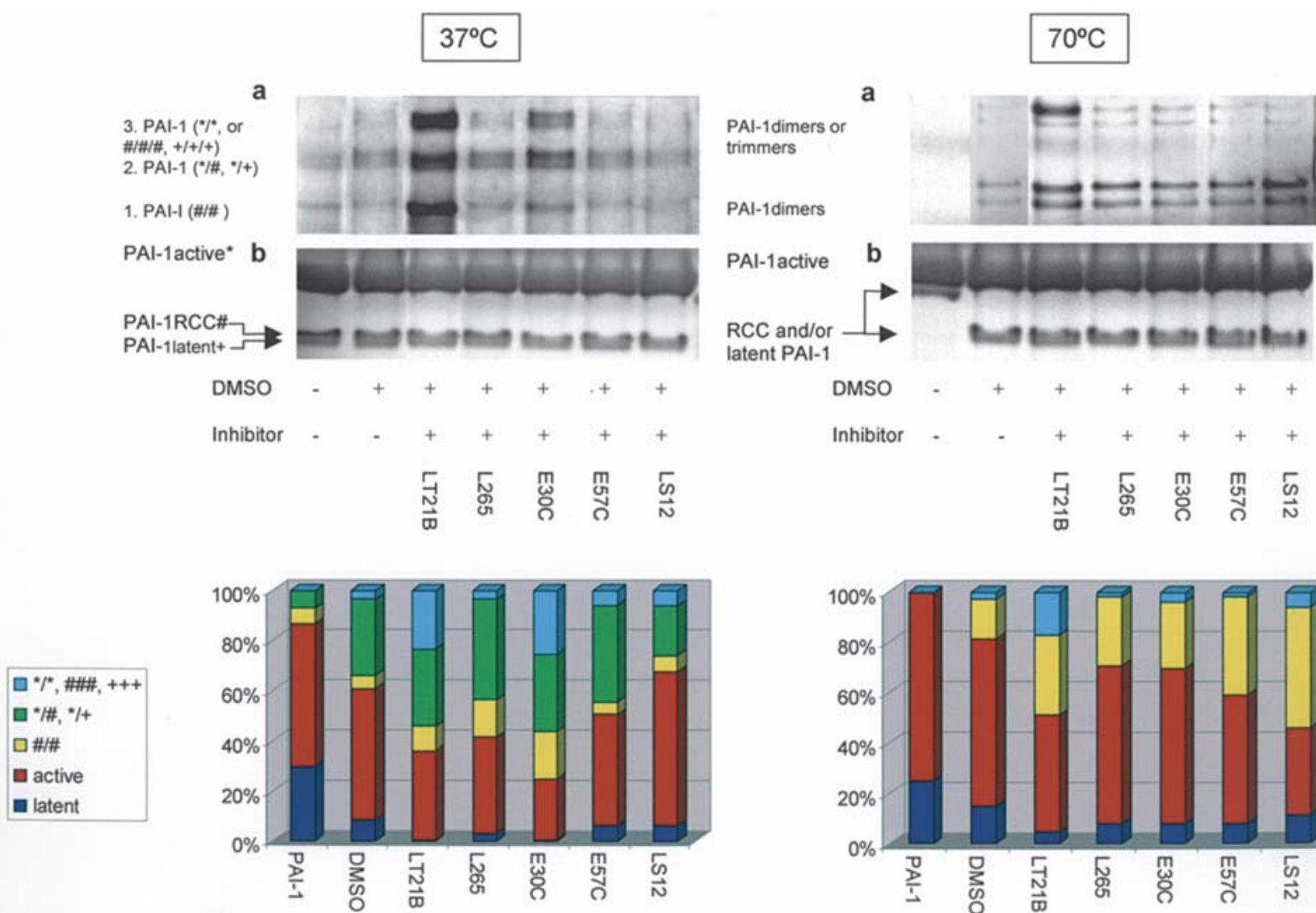

Figure 4. PAI-1 incubated with its inhibitors for 30 min followed by incubation with SDS loading buffer in different temperatures. a, silver stain; b, colloidal Coomassie stain of SDS-PAGE gels. Molecular weight of protein bands indicates polymers of PAI-1: 1, two RCC molecules; 2, active and latent or active and RCC; 3, two molecules of active PAI-1, or three molecules of RCC or latent PAI-1. Heating samples to $70^{\circ} \mathrm{C}$ caused a drastic reduction of heterogenic polymers, e.g. dimers of latent and active or RCC and active PAI-1s. Color bars represents fractions of each PAI-1 form based on their relative color band intensity.

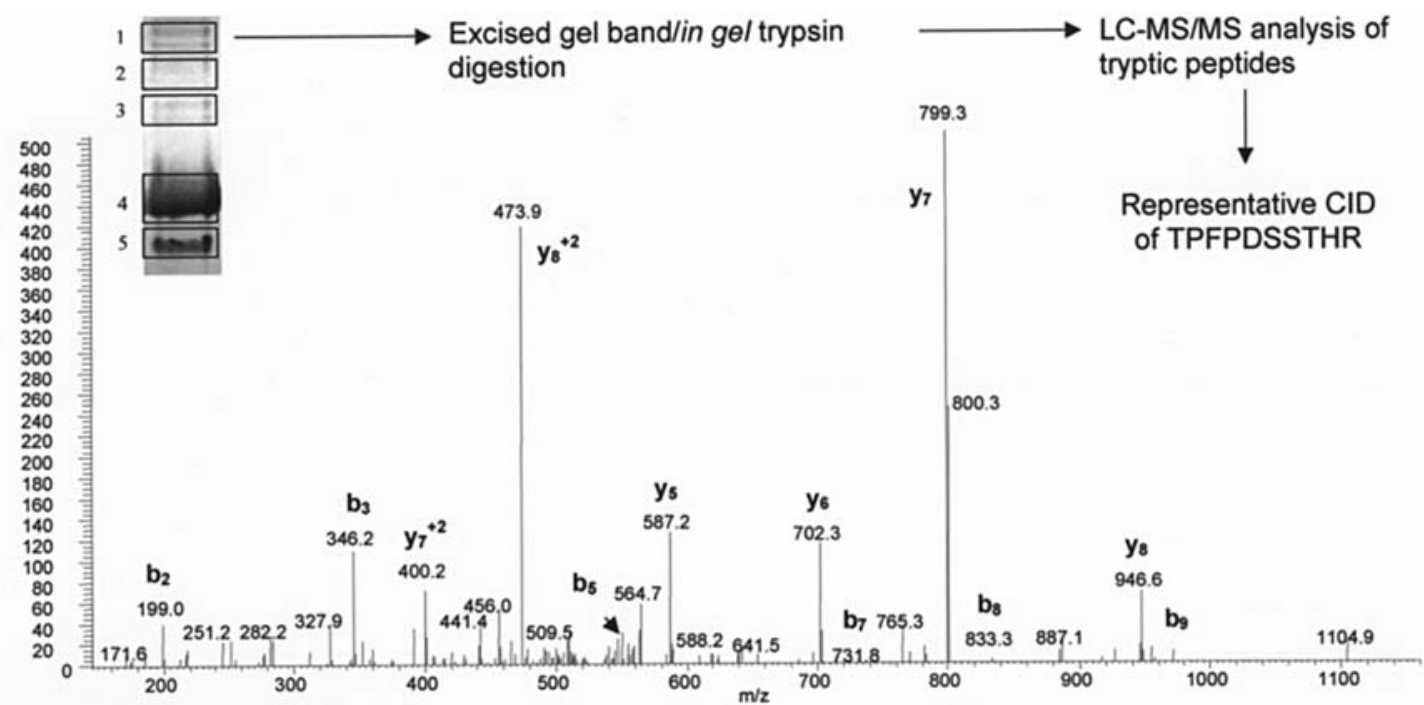

Figure 5. Example of peptide analysis by mass spectroscopy. Excised from gel band proteins were digested with trypsin and analyzed on LC-MS/MS. Mass spectrogram shows, among others, the TPFPDSSTHR peptide. For example, the molecular weights of the following fragments are: FPDSSTHR of MW 946.6 (946.9); PDSSTHR of MW 799.3 (799.8); DSSTHR of MW 702.3 (702.6); SSTHR of MW 587.2 (587.6) (numbers in parentheses indicate theoretical molecular weight). This ten amino-acid peptide is split for $y$ and $b$ fragments. For example, if the $y_{8}$ (FPDSSTHR, MW 946.6) fragment $b_{2}$ shall be detected as well (TP, MW 199) yielding MW of 1144.2 of the entire peptide TPFPDSSTHR (45).

Protein identification by peptide sequencing using liquid chromatography-tandem mass spectrometer. A total of six bands were excised from the gel. As shown in Fig. 5, bands marked as 1,2 , and 3 contain two distinct protein bands which were too close to separate. Also, below band 5, very weak bands (not shown in Fig. 5) were detected and analyzed. As shown in Table III, bands 1-5 were identified as PAI-1 and no other contaminating proteins were detected. 
Table III. Sequence of peptides extracted from different bands of monomer and polymers of PAI-1.

\begin{tabular}{|c|c|c|c|}
\hline Band(s) & Protein accession no. & Peptides sequenced in PAI-1 molecule & $\%$ of sequence coverage \\
\hline Bands 1 & P05121 & $\begin{array}{l}24-33,42-51,104-111,146-154,157-164,169-177 \\
200-209,215-230,301-310,311-323\end{array}$ & 23 \\
\hline Bands 2 & P05121 & $\begin{array}{l}24-33,42-51,104-111,146-154,157-164,169-177, \\
178-185,215-230,301-310,311-323\end{array}$ & 25 \\
\hline Bands 3 & P05121 & $\begin{array}{l}24-33,42-51,104-111,112-124,128-135,146-154, \\
169-177,178-185,200-209,215-230,301-310,311- \\
323,370-379\end{array}$ & 33 \\
\hline Band 4 & P05121 & $\begin{array}{l}24-33,42-51,104-111,128-135,139-141,146-154, \\
157-164,169-177,178-185,190-199,200-209,211- \\
214,215-230,301-310,311-323,387-402\end{array}$ & 37 \\
\hline Band 5 & P05121 & $\begin{array}{l}42-51,100-103,104-111,139-141,146-154,157-164 \\
200-209,211-214,215-230,258-266,295-300,301- \\
310,311-323,387-402\end{array}$ & 33 \\
\hline
\end{tabular}

Full sequence of PAI-1

\begin{tabular}{|c|c|c|c|c|c|c|c|}
\hline & 1 & 11 & 21 & 31 & 41 & 51 & \\
\hline & $\mid$ & 1 & $\mid$ & 1 & | & 1 & \\
\hline 1 & MQMSPALTCL & VLGLALVFGE & GSAVHHPPSY & VAHLASDFGV & RVFQQVAQAS & KDRNVVFSPY & 60 \\
\hline 61 & GVASVLAMLQ & LTTGGETQQQ & IQAAMGFKID & DKGMAPALRH & LYKELMGPWN & KDEISTTDAI & 120 \\
\hline 121 & FVQRDLKLVQ & GFMPHFFRLF & RSTVKQVDFS & EVERARFIIN & DWVKTHTKGM & ISNLLGKGAV & 180 \\
\hline 181 & DQLTRLVLVN & ALYFNGQWKT & PFPDSSTHRR & LFHKSDGSTV & SVPMMAQTNK & FNYTEFTTPD & 240 \\
\hline 241 & GHYYDILELP & YHGDTLSMFI & AAPYEKEVPL & SALTNILSAQ & LISHWKGNMT & RLPRLLVLPK & 300 \\
\hline 301 & FSLETEVDLR & KPLENLGMTD & MFRQFQADFT & SLSDQEPLHV & AQALQKVKIE & VNESGTVASS & 360 \\
\hline 361 & STAVIVSARM & APEEI IMDRP & FLFVVRHNPT & GTVLFMGQVM & $\mathrm{EP}$ & & \\
\hline
\end{tabular}

Table IV. Sequence of peptides in $30 \mathrm{~S}$ and $50 \mathrm{~S}$ ribosomal proteins.

\begin{tabular}{llcccr}
\hline Protein accession no. & Protein name & Theoretical mass & Observed mass & Peptide & Sequence \\
\hline P02359 & $\begin{array}{l}\text { 30S } \\
\text { ribosomal } \\
\text { protein S7 }\end{array}$ & 1274.6 & 1274.3 & $120-131$ & LANELSDAAENK \\
& & & & & \\
& & & & $3-12$ & TFTAKPETVK \\
P02410 & 1121.6 & 1121.5 & 1482.2 & $73-85$ & VYYHHTGHIGGIK \\
& ribosomal & 1481.7 & 1211.7 & $86-95$ & QATFEEmIAR \\
\hline
\end{tabular}

$\mathrm{m}$, oxidized methionine.

Below latent and RCC, PAI-1's fine band was detected that includes minute amounts of two different proteins identified as $30 \mathrm{~S}$ ribosomal protein $\mathrm{S} 7$ of $19.8 \mathrm{kDa}$, and $50 \mathrm{~S}$ ribosomal protein L13 16.0 kDa of E. coli (Table IV).

Docking. It has been reported that inhibition of PAI-1 was induced by the binding of an antibody into PAI-1, followed by the rapid conversion of PAI-1 into its latent form (45). Our experiments showed that PAI-1 small molecule inhibitors arrest PAI-1 in its active conformation rather than speeding its conversion. The most rational explanation of PAI-1 inhibition would be the binding of inhibitors on the RCL, most likely close to the $\mathrm{P} 1 / \mathrm{P} 1$ ' positions, which are critical for uPA/PAI-1 complex formation. 
Table V. Best scored PAI-1 inhibitors. ${ }^{\mathrm{a}}$

\begin{tabular}{|c|c|c|c|c|}
\hline Inhibitor & Structure & Score & H-bonds & $\begin{array}{c}\text { Hydrophobic } \\
\text { complementarity }\end{array}$ \\
\hline E30C-keto & & 75.4 & 6 & 62.9 \\
\hline E30C-enol & & 62.2 & 4 & 87.3 \\
\hline LS12(+) & & 54.8 & 12 & 18.3 \\
\hline LT21B $(+)$ & & 54.6 & 10 & 27.4 \\
\hline L265 & & 52.6 & 2 & 61.5 \\
\hline $\mathrm{LT} 21 \mathrm{Bb}(+)$ & & 50.1 & 12 & 29.1 \\
\hline E57C-enol & & 46.2 & 4 & 60.0 \\
\hline LT21B & & 43.6 & 6 & 46.2 \\
\hline LS12 & & 41.8 & 4 & 43.1 \\
\hline E57C-keto & & 40.1 & 2 & 59.0 \\
\hline
\end{tabular}

${ }^{\mathrm{a}}$ Hydrogen atoms were generated by the program where necessary.

The RCL of PAI-1 in its active form is highly flexible. Trying to model the conformation of this loop in the active monomeric form of PAI-1 would add uncertainties to the results of the computational docking using the vicinity of this loop as the target-binding site. Thus, we used the structure $1 \mathrm{~B} 3 \mathrm{~K}$ of PBD entry of PAI-1 that in the X-ray molecular structure shows typical to serpins RCL/s6A polymerization (46). Two molecules $(\mathrm{A}, \mathrm{C})$ that form a dimer were used as targets for docking the enol- and keto-forms of curcuminoids, as well as charged and non-charged forms of the other inhibitors. For all inhibitors, different configurations and conformations were tested and evaluated by the consensus scoring. It was found that all inhibitors bind in the proximity of P1, P1'. As shown in Table V, the inhibitors with the highest theoretical affinity were E30C, LS12(+) and LT21B(+), while L265 and E57C showed lower scores. Also, as shown in Fig. 6, two distinct binding sites of PAI-1 inhibitors were detected. One site for positively charged inhibitors, which link the RCL of molecule A with turn Ala26 $\rightarrow$ Asn 31 extending from helix hA to strand s1B of PAI-1's molecule C. A second site for non-charged molecules, which preferred to bind in the proximity of $\mathrm{s} 6 \mathrm{~A}, \mathrm{~s} 3 \mathrm{C}$ and $\mathrm{s} 4 \mathrm{C}$ strands of chain $\mathrm{C}$ and the RCL loop of chain A. In all cases, inhibitors are located in a position in which they link chains $\mathrm{A}$ and $\mathrm{C}$ in such a fashion that they can contribute to stabilization of the dimer interface and shift the equilibrium toward polymerization of PAI-1 molecules. The docked orientation of the highest scoring inhibitors identified by DrugScore are somewhat different from that favored by the internal scoring system of the SLIDE program. However, a general pattern of interactions could be observed in all cases supporting the proposed model of PAI-1 inhibition and polymerization. For consistency and clarity of presentation, only the docked orientations favored by SLIDE scoring functions are presented in this paper.

\section{Discussion}

Inhibition activity or, as it is called by some, PAI-1's neutralizing activity has been previously reported $(22,23,46-48)$. Antibody or organic chemicals binding to the RCL achieved this effect which, for obvious reasons, must impair the ability of PAI-1 to bind to UPA or tPA. However, antibodies and organochemicals that bind to parts of PAI- 1 other than the reactive site loop can also inactivate PAI-1. The MA-33B8 antibody accelerated the conversion of PAI-1 into its latent form upon binding, reaching a speed 4,000-fold faster than the normal rate of conversion. The authors concluded that MA-33B8 binding to PAI-1 promotes the insertion of the RCL into $B$-sheet A, yet the exact mechanism remained unclear (45). Egelund et al suggested that the various PAI-1 neutralizers have overlapping, but not identical, binding sites in the region around $\alpha$-helices $\mathrm{D}$ and $\mathrm{E}$ and $\beta$-strand $1 \mathrm{~A}$. This part is known to act as a flexible joint when $B$-sheet $\mathrm{A}$ opens and part of the RCL is inserted as $B$-strand 4A during conversion into the latent form (46). Bodker et al mapped the PAI-1 molecule to localize site responsible for the inhibitory activity upon binding of different agents. They concluded that the site is localized in $\alpha$-helix $\mathrm{C}$ and the loop connecting $\alpha$-helix $\mathrm{I}$ and $\beta$-strand $5 \mathrm{~A}$. This site is opposite to $B$-sheet $\mathrm{A}$ and distant from the flexible joint region and RCL (49). As a consequence, Debrock and Declerck proposed two distinct mechanisms of PAI-1 inactivation. One is the direct interaction with the reactive-site loop; the second facilitates induced conversion into the latent conformation of PAI-1 (22).

Our data show a third possibility; the binding of inhibitors at the RCL and arrest of the PAI-1 molecule in its active form. Any chemicals that bind to PAI-1 in the proximity of the P1, P1' position on the RCL will hinder the binding of PAI-1 to uPA or tPA. Also, a bulge of added chemical on that loop would make the insertion of the RCL between B-strand $3 \mathrm{~A}$ and $5 \mathrm{~A}$ of PAI-1 during its conversion from active to latent form more difficult, as in the case of NBD P9 PAI-1. Space interference of the covalently bound NBD molecule into the mutated P9 position in the RCL created a mutant, which was almost as reactive as the wild-type, but was considerably more stable. The half-life of the active form was extended from $2 \mathrm{~h}$ of wPAI- 1 to $24 \mathrm{~h}$ in the case of NBD P9 PAI-1 (50).

Serpins, in general, are able to link their RCL to a $B$-sheet of another molecule, forming thermodynamically stable and inactive complexes (42). In all reported cases, there is support for three different types of loop-sheet polymerization: i) polymerization by the RCL of one molecule inserting as s4A 


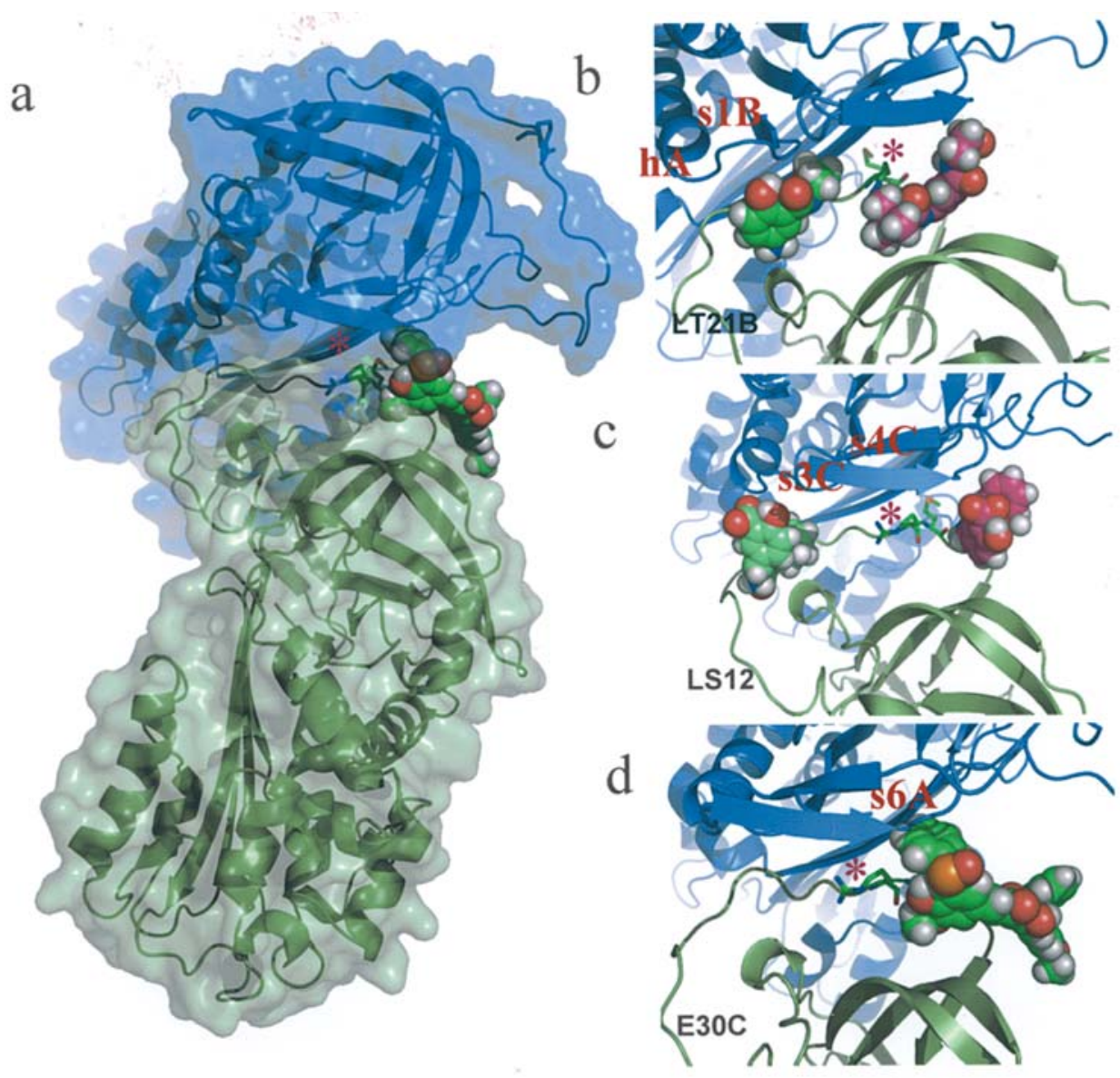

Figure 6. a, ribbon model of A (light green) and C (light blue) chains of active PAI-1 with semitransparent molecular surface (atomic coordinates for the dimer structure from 1B3K). Position of P1/P1' site indicated by magenta asterisk with Arg-Met amino acids shown as a stick model. A space-filing model indicates the calculated position of the E30C inhibitor. b and c, ribbon model of A and C chains of PAI-1 with the most probable position of the LT21B and LS12 inhibitors shown as a space-filing model. Hydrogens are shown in white, nitrogens in blue, oxygens in red. Carbons in magenta indicate non-charged molecules, carbons in green indicate positively charged inhibitors and top scored molecules. d, position of top scored E30C inhibitor.

of another molecule (51); ii) the RCL of one molecule can insert as $\mathrm{S} 1 \mathrm{C}$ in another molecule; iii) the RCL of one molecule can form hydrogen bonds to s6A of another molecule and thus form an additional strand s7A (46). The evidence for the latter type of polymerization is provided by structural studies of active PAI-1 (PDB entry 1B3K) (37).

Induction of PAI-1 polymerization has limited literature, but it was detected as induced by small organochemical ligands or by incubation in low $\mathrm{pH}(42,46)$. Our data support a very similar model of inhibition linked to polymerization; however, our inhibitors are most likely attached at different regions of the protein molecule than previously reported. Our results suggest that tested organochemicals further neutralize PAI-1 activity by linking both chains of PAI-1 in the proximity of the P1, P1' site, blocking at least one RCL in the dimer. It is plausible that these molecules also bind to the monomeric form of PAI-1 and inhibit its activity. Additional effects of PAI-1 inactivation could be related to the immobilization of the $\mathrm{s} 3 \mathrm{C}$ and $\mathrm{s} 4 \mathrm{C}$ regions of PAI-1 called GATE. It has been shown that mutations in this part of the PAI-1 molecule could inactivate it $(52,53)$. Clearly, some of the PAI-1 inhibitors that we tested could be binding there.

$\mathrm{RCL} / \mathrm{s} 6 \mathrm{~A}$ type polymerization has been reported for different serpins, but the possibility of polymerization of active/latent, active/RCC, latent/latent, and RCC/RCC forms of PAI-1, which are clearly visible on SDS-PAGE gels, is novel to our findings. The formation of heterogenic complexes of active and latent or RCC PAI-1 forms is quite possible, as strands s3C and s4C, as well as turn Ala26 $\rightarrow$ Asn31 that extends from helix hA to strand s1B, are accessible to inhibitors after PAI-1 conversion into latent or RCC forms $(18,54)$. The formation of RCL cleaved (RCC) and latent PAI-1 forms is also quite credible, as crystal structure data show that the cleaved RCL can still access stable regions of s3C, s4C, helix $\mathrm{hA}$ and $\mathrm{s} 1 \mathrm{~B}$, as well as the part cleaved at the P1/P1' RCL. A cleaved fragment of the RCL loop (up-stream of P1') could serve as a linker between two different PAI-1 molecules. This fragment is in an almost identical position in the X-ray structure (PDB entry 1A7C) as the intact RCL fragment of the active form (54). It must be emphasized that we were not able to perform any molecular modeling on this type of molecule in the absence of any suitable structures and therefore cannot confirm our assumptions.

With respect to the functional inhibition of PAI-1-uPA interaction by the small molecule organochemicals, the difference in the effectiveness of individual compounds to prolong the active conformational state of PAI-1 versus functionally inhibiting it, could be due to several mechanisms. 
Whereas binding of the organochemical molecule to the RCL region of PAI-1 may be sufficient to arrest the PAI-1 in an active form, it is possible that the bound compound may only partially prevent the interaction of PAI-1 with uPA. This mechanism of inhibition of PAI-1 by the small organochemicals may depend on the chemical characteristics of the compound, including its size, hydrophobicity or charge state. This mechanism of action may underlie the observed differences in rank order of potency of the various compounds when the residual uPA assay versus the spectral shift assay is used as the endpoint.

If the organochemical bound to the RCL region causes a reduction in the affinity of the serpin for $\mathrm{UPA}$, it might explain why a partial inhibition was observed within the dose-range that the compounds were tested (Table II). It is also possible that the compounds undergo association and dissociation from the RCL region of PAI-1 during the course of the functional assay, thus exhibiting only partial inhibition of uPA by PAI-1, even at high concentrations of the compound. It is also possible that binding of the organochemical compound to the RCL region and the subsequent elevated polymerization of PAI-1 is an important mechanism for the observed functional inhibition of PAI-1's interaction with uPA. The time required for the polymerization of PAI-1 to occur after binding of the organochemical compound may be longer than the incubation utilized in the functional uPA assay. It must also be emphasized that the functional assay of PAI-1 reported in Table II is an indirect assay in which residual uPA remaining after inhibition by active PAI-1 is measured as the endpoint for the PAI-1 activity in the presence of various concentrations of small molecule organochemical inhibitors. Another limitation of this assay is that some compounds, such as E57C, have an intense yellow-red color, which can quench/interfere with the fluorescent emission of the product of the uPA reaction. It is also possible that some of these small organic compounds may inhibit UPA independent of PAI-1, although this is likely to happen at much higher concentrations than are required to inhibit PAI-1. For example, at $100 \mu \mathrm{M}$, E30C and LS12 both reduced uPA activity by $20-30 \%$ (data not shown), but their inhibitory activity towards PAI-1 was largely reduced at $10 \mu \mathrm{M}$, a dose at which they had no direct effect on uPA. Due to these considerations, uPA inhibition may not be the best functional endpoint to measure functional inhibition of PAI-1 by these small molecule inhibitors.

This study provides evidence of inhibiting PAI-1 activity by small organic chemicals. The chemicals that we tested most likely bind to the reactive center loop of PAI-1. Binding in this region can also induce the polymerization of PAI-1 and further inactivate PAI-1 by encapsulating the RCL fragment, critical to its function, inside of formed PAI-1/PAI-1 polymers.

\section{Acknowledgments}

The authors (J.J., E.S.J.) wish to thank Dr Richard Hart for his unfailing support, chemicals and valuable discussions. We thank Dr Gerhard Klebe (University of Marburg, Germany) for generously providing DrugScore for our use. We also thank OpenEye Scientific Software, Inc. for making their software available to us free of charge. This work was supported in part by grants from NIH CA90524, CA109625; and Frank D. Stranahan Endowment Fund for Oncological Research.

\section{References}

1. Duffy MJ: The urokinase plasminogen activator system: role in malignancy. Curr Pharm Des 10: 39-49, 2004.

2. De Bock CE and Wang Y: Clinical significance of urokinasetype plasminogen activator receptor (uPAR) expression in cancer. Med Res Rev 24: 13-39, 2004.

3. Mazar AP, Henkin J and Goldfarb RH: The urokinase plasminogen activator system in cancer: implications for tumor angiogenesis and metastasis. Angiogenesis 3: 15-32, 1999.

4. Alfano M, Sidenius N, Blasi F and Poli G: The role of urokinasetype plasminogen activator (uPA)/uPA receptor in HIV-1 infection. J Leukoc Biol 74: 750-756, 2003.

5. Dellas C and Loskutoff DJ: Historical analysis of PAI-1 from its discovery to its potential role in cell motility and disease. Thromb Haemost 93: 631-640, 2005.

6. Chorostowska-Wynimko J, Skrzypczak-Jankun E and Jankun J: Plasminogen activator inhibitor type-1: its structure, biological activity and role in tumorigenesis (Review). Int J Mol Med 13: 759-766, 2004.

7. Agirbasli M: Pivotal role of plasminogen-activator inhibitor 1 in vascular disease. Int J Clin Pract 59: 102-106, 2005.

8. Yamamoto $\mathrm{K}$ and Saito H: A pathological role of increased expression of plasminogen activator inhibitor-1 in human or animal disorders. Int J Hematol 68: 371-385, 1998.

9. Hasenstab D, Lea H and Clowes AW: Local plasminogen activator inhibitor type 1 overexpression in rat carotid artery enhances thrombosis and endothelial regeneration while inhibiting intimal thickening. Arterioscler Thromb Vasc Biol 20: 853-859, 2000 .

10. Hornebeck W, Emonard H, Monboisse JC and Bellon G: Matrix-directed regulation of pericellular proteolysis and tumor progression. Semin Cancer Biol 12: 231-241, 2002.

11. Stephens RW, Brunner N, Janicke F and Schmitt M: The urokinase plasminogen activator system as a target for prognostic studies in breast cancer. Breast Cancer Res Treat 52: 99-111, 1998.

12. Duffy MJ: Proteases as prognostic markers in cancer. Clin Cancer Res 2: 613-618, 1996

13. Swiercz R, Skrzypczak-Jankun E, Merrell MM, Selman SH and Jankun J: Angiostatic activity of synthetic inhibitors of urokinase type plasminogen activator. Oncol Rep 6: 523-526, 1999.

14. Swiercz R, Keck RW, Skrzypczak-Jankun E, Selman SH and Jankun J: Recombinant PAI-1 inhibits angiogenesis and reduces size of LNCaP prostate cancer xenografts in SCID mice. Oncol Rep 8: 463-470, 2001.

15. Chorostowska-Wynimko J, Swiercz R, Skrzypczak-Jankun E, Wojtowicz A, Selman SH and Jankun J: A novel form of the plasminogen activator inhibitor created by cysteine mutations extends its half-life: relevance to cancer and angiogenesis. Mol Cancer Ther 2: 19-28, 2003.

16. Stefansson S, McMahon GA, Petitclerc E and Lawrence DA: Plasminogen activator inhibitor-1 in tumor growth, angiogenesis and vascular remodeling. Curr Pharm Des 9: 1545-1564, 2003.

17. McMahon GA, Petitclerc E, Stefansson S, Smith E, Wong MK, Westrick RJ, Ginsburg D, Brooks PC and Lawrence DA: Plasminogen activator inhibitor-1 regulates tumor growth and angiogenesis. J Biol Chem 276: 33964-33968, 2001.

18. Tucker HM, Mottonen J, Goldsmith EJ and Gerard RD: Engineering of plasminogen activator inhibitor-1 to reduce the rate of latency transition. Nat Struct Biol 2: 442-445, 1995.

19. Mottonen J, Strand A, Symersky J, Sweet RM, Danley DE, Geoghegan KF, Gerard RD and Goldsmith EJ: Structural basis of latency in plasminogen activator inhibitor-1. Nature 355: 270-273, 1992 .

20. Berkenpas MB, Lawrence DA and Ginsburg D: Molecular evolution of plasminogen activator inhibitor-1 functional stability. EMBO J 14: 2969-2977, 1995.

21. Steinmetzer T: Synthetic urokinase inhibitors as potential antitumor drugs. Drugs 6: 138-146, 2003.

22. Debrock S and Declerck PJ: Neutralization of plasminogen activator inhibitor-1 inhibitory properties: identification of two different mechanisms. Biochim Biophys Acta 1337: 257-266, 1997.

23. Pedersen KE, Einholm AP, Christensen A, Schack L, Wind T, Kenney JM and Andreasen PA: Plasminogen activator inhibitor-1 polymers, induced by inactivating amphipathic organochemical ligands. Biochem J 372: 747-755, 2003. 
24. Komissarov AA, Andreasen PA, Bodker JS, Declerck PJ, Anagli JY and Shore JD: Additivity in effects of vitronectin and monoclonal antibodies against alpha-helix $\mathrm{F}$ of plasminogen activator inhibitor- 1 on its reactions with target proteinases. J Biol Chem 280: 1482-1489, 2005.

25. Strandberg L, Karolin J, Johansson LB, Fa M, Aleshkov S and Ny T: Fluorescence studies on plasminogen activator inhibitor 1 : reactive centre cysteine mutants remain active after fluorophore attachment. Thromb Res 76: 253-267, 1994.

26. Pabon HJJ: A synthesis of curcumin and related compounds. Rec Trav Chim Pays-Bas 83: 379-385, 1964.

27. Di Santo R, Costi R, Artico M, Ragno R, Greco G, Novellino E, Marchand C and Pommier Y: Design, synthesis and biological evaluation of heteroaryl diketohexenoic and diketobutanoic acids as HIV-1 integrase inhibitors endowed with antiretroviral activity. Farmaco 60: 409-417, 2005

28. Robinson TP, Hubbard RB, Ehlers TJ, Arbiser JL, Goldsmith DJ and Bowen JP: Synthesis and biological evaluation of aromatic enones related to curcumin. Bioorg Med Chem 13: 4007-4013, 2005.

29. Komissarov AA, Declerck PJ and Shore JD: Protonation state of a single histidine residue contributes significantly to the kinetics of the reaction of plasminogen activator inhibitor-1 with tissuetype plasminogen activator. J Biol Chem 279: 23007-23013, 2004.

30. Olson ST, Bock PE, Kvassman J, Shore JD, Lawrence DA, Ginsburg D and Bjork I: Role of the catalytic serine in the interactions of serine proteinases with protein inhibitors of the serpin family. Contribution of a covalent interaction to the binding energy of serpin-proteinase complexes. J Biol Chem 270: 30007-30017, 1995.

31. Tunon P and Johansson KE: Yet another improved silver staining method for the detection of proteins in polyacrylamide gels. J Biochem Biophys Methods 9: 171-179, 1984.

32. Basrur V, Yang F, Kushimoto T, Higashimoto Y, Yasumoto K, Valencia J, Muller J, Vieira WD, Watabe H, Shabanowitz J, Hearing VJ, Hunt DF and Appella E: Proteomic analysis of early melanosomes: identification of novel melanosomal proteins. J Proteome Res 2: 69-79, 2003.

33. Schnecke V and Kuhn LA: Virtual screening with solvation and ligand-induced complementarity. Perspect Drug Disc Des 20: $171-190,2000$

34. Zavodszky MI, Sanschagrin PC, Korde RS and Kuhn LA: Distilling the essential features of a protein surface for improving protein-ligand docking, scoring, and virtual screening. J Comput Aided Mol Des 16: 883-902, 2002.

35. Schnecke V and Kuhn LA: Database screening for HIV protease ligands: the influence of binding-site conformation and representation on ligand selectivity. Proceedings of the Seventh International Conference in Intelligent Systems for Molecular Biology, AAAI Press, 242-251, 1999.

36. Gohlke H, Hendlich M and Klebe G: Knowledge-based scoring function to predict protein-ligand interactions. J Mol Biol 295: 337-356, 2000.

37. Sharp AM, Stein PE, Pannu NS, Carrell RW, Berkenpas MB, Ginsburg D, Lawrence DA and Read RJ: The active conformation of plasminogen activator inhibitor 1 , a target for drugs to control fibrinolysis and cell adhesion. Structure Fold Des 7: 111-118, 1999.

38. Guex N and Peitsch MC: SWISS-MODEL and the SwissPdbViewer: an environment for comparative protein modeling. Electrophoresis 18: 2714-2723, 1997.

39. Baker NA, Sept D, Joseph S, Holst MJ and McCammon JA: Electrostatics of nanosystems: application to microtubules and the ribosome. Proc Natl Acad Sci USA 98: 10037-10041, 2001 .
40. Aleshkov SB, Fa M, Karolin J, Strandberg L, Johansson LB, Wilczynska $M$ and Ny T: Biochemical and biophysical studies of reactive center cleaved plasminogen activator inhibitor type 1 . The distance between P3 and P1' determined by donor-donor fluorescence energy transfer. J Biol Chem 271: 21231-21238, 1996.

41. Olson ST, Swanson R, Day D, Verhamme I, Kvassman J and Shore JD: Resolution of Michaelis complex, acylation, and conformational change steps in the reactions of the serpin, plasminogen activator inhibitor-1, with tissue plasminogen activator and trypsin. Biochemistry 40: 11742-11756, 2001.

42. Zhou A, Faint R, Charlton P, Dafforn TR, Carrell RW and Lomas DA: Polymerization of plasminogen activator inhibitor-1. J Biol Chem 276: 9115-9122, 2001.

43. Wang H, Pap S and Wiman B: Inactivation of antiplasmin at low pH: evidence for the formation of latent molecules. Thromb Res 114: 301-306, 2004.

44. Wang Q and Shaltiel S: Distal hinge of plasminogen activator inhibitor-1 involves its latency transition and specificities toward serine proteases. BMC Biochem 4: 1-15, 2003.

45. Verhamme I, Kvassman JO, Day D, Debrock S, Vleugels N, Declerck PJ and Shore JD: Accelerated conversion of human plasminogen activator inhibitor-1 to its latent form by antibody binding. J Biol Chem 274: 17511-17517, 1999.

46. Egelund R, Einholm AP, Pedersen KE, Nielsen RW, Christensen A, Deinum J and Andreasen PA: A regulatory hydrophobic area in the flexible joint region of plasminogen activator inhibitor-1, defined with fluorescent activity-neutralizing ligands. Ligand-induced serpin polymerization. J Biol Chem 276: 13077-13086, 2001.

47. Gils A, Lu J, Aertgeerts K, Knockaert I and Declerck PJ: Identification of positively charged residues contributing to the stability of plasminogen activator inhibitor 1. FEBS Lett 415: 192-195, 1997.

48. Naessens D, Gils A, Compernolle G and Declerck PJ: Elucidation of the epitope of a latency-inducing antibody: identification of a new molecular target for PAI-1 inhibition. Thromb Haemost 90: $52-58,2003$.

49. Bodker JS, Wind T, Jensen JK, Hansen M, Pedersen KE and Andreasen PA: Mapping of the epitope of a monoclonal antibody protecting plasminogen activator inhibitor-1 against inactivating agents. Eur J Biochem 270: 1672-1679, 2003.

50. Shore JD, Day DE, Francis-Chmura AM, Verhamme I, Kvassman J, Lawrence DA and Ginsburg D: A fluorescent probe study of plasminogen activator inhibitor-1. Evidence for reactive center loop insertion and its role in the inhibitory mechanism. J Biol Chem 270: 5395-5398, 1995.

51. Katsumi TA, Aizawa K, Kuroiwa Y, Saito K, Kurata Y, Ii Y, Okunaka T, Konaka C and Kato H: Photodynamic therapy with a diode laser for implanted fibrosarcoma in mice employing mono-L-aspartyl chlorin E6. Photochem Photobiol 64: 671-675, 1996.

52. Wardell MR, Chang WS, Bruce D, Skinner R, Lesk AM and Carrell RW: Preparative induction and characterization of L-antithrombin: a structural homologue of latent plasminogen activator inhibitor-1. Biochemistry 36: 13133-13142, 1997.

53. Vleugels N, Leys J, Knockaert I and Declerck PJ: Effect of stabilizing versus destabilizing interactions on plasminogen activator inhibitor-1. Thromb Haemost 84: 871-875, 2000.

54. Aertgeerts K, De Bondt HL, De Ranter CJ and Declerck PJ: Mechanisms contributing to the conformational and functional flexibility of plasminogen activator inhibitor-1. Nat Struct Biol 2: 891-897, 1995 . 